\title{
SATURABLE ABSORPTION OF DYES EXCITED TO THE LONG-WAVELENGTH REGION OF THE $S_{0}-S_{1}$ ABSORPTION BAND
}

\author{
W. BLAU, W. DANKESREITER and A. PENZKOFER \\ Naturwissenschaftliche Fakultät II - Physik, Universität Regensburg, 8400 Regensburg, FRG
}

Received 12 September 1983

\begin{abstract}
Organic dyes are excited with intense picosecond ruby laser pulses to the long-wavelength wing of the $S_{0}-S_{1}$ transition beyond the fluorescence emission peak. A model is presented where only a fraction of the molecules interacts with laser light and the excitation terminates in the $S_{1}$ equilibrium level. Bleaching is readily observed but laser action and amplified spontaneous emission are reduced.
\end{abstract}

\section{Introduction}

The dynamics of $S_{1}-S_{0}$ relaxation of dye solutions is determined by the solute, the solvent, and the excitation source. The frequency of excitation influences the relaxation mechanisms: Promotion of molecules to higher singlet states $S_{n}(n \geqslant 2)$ opens decay channels mainly to the $S_{1}$ state, to the $S_{0}$ state, to triplet states and to molecular decomposition $[1,2]$. For molecules tending to form excitedstate conformers the $\mathrm{S}_{1}-\mathrm{S}_{0}$ decay mechanism depends on the excess energy of the excitation compared to the $\mathrm{S}_{1}\left(v^{\prime}=0\right)-\mathrm{S}_{0}\left(v^{\prime \prime}=0\right)$ (zero vibration) transition energy. The excess energy facilitates excited-state isomer formation and opens new decay channels [3-6]. The pump light intensity plays a decisive role in $S_{1}-S_{0}$ relaxation when intense picosecond pulses are used for $S_{1}$ state population: amplified spontaneous emission enhances the depopulation of the $S_{1}$ state $[7,8]$, excited-state absorption promotes molecules to higher-lying states $[9,10]$ and initiates new decay channels.

The saturable absorption of dyes excited with picosecond laser pulses of frequency $\nu_{L}$ less than the frequency of optimum fluorescence emission $\nu_{\mathrm{F}, \mathrm{m}}$ is studied in this paper. A realistic model for the absorption and relaxation processes is presented. In contrast to short-wavelength excitation $\left(\nu_{\mathrm{L}} \gtrsim \nu_{\mathrm{A}, \mathrm{m}}, \nu_{\mathrm{A}, \mathrm{m}}\right.$ frequency of maximum $\mathrm{S}_{0}-\mathrm{S}_{1}$ ab- sorption), here the excitation terminates in the temporal equilibrium position of the $S_{1}$ state from where the fluorescence emission originates. Only thermally excited molecules or molecules in the long-wavelength tail of the inhomogeneous $S_{0}-S_{1}$ transition frequency distribution can interact with the pump laser. As a consequence the bleaching of dyes is readily observed (only a fraction of molecules absorbs at the long wavelength side) while amplified spontaneous emission and laser action is reduced (re-absorption of fluorescence emission with $\nu>\nu_{\mathrm{L}}$ due to ground-state population). The effects of spectral cross-relaxation in the $S_{0}$ band on the absorption dynamics will be discussed.

Experiments are performed on some organic dyes with a passively mode-locked ruby laser. The experimental results confirm the theoretical considerations. Some aspects of pulse propagation in fluorescing dye media have been reported previously [11-16]. Especially dyes with slow solute-solvent relaxation have been studied in refs. [11-15].

\section{Theory}

The absorption spectrum of dye solutions is rather complex. The $S_{0}$ ground-state levels are thermally populated, the $S_{0}-S_{1}$ frequency spacing 
is inhomogeneously broadened due to irregular actions of the solvent, and a manifold of transitions between $S_{0}$ and $S_{1}$ rovibrational levels are possible at a fixed laser frequency $\nu_{\mathrm{L}}$. Additionally each transition is homogeneously broadened by phase changes and finite population lifetimes. Reduction of this multi-transition problem to a fourlevel system was achieved in ref. [17] for $\nu_{\mathrm{L}} \gtrsim \nu_{\mathrm{A}, \mathrm{m}}$. Necessary conditions for the reduction are that either the spectral cross-relaxation is fast compared to the pump pulse duration or approximately all molecules take part in the absorption with nearly equal absorption coefficients. The four-level description may be applied approximately to the frequency region $\nu_{\mathrm{A}, \mathrm{m}}>\nu_{\mathrm{L}}>\nu_{\mathrm{F}, \mathrm{m}}$. It breaks down for $\nu_{L} \leqslant \nu_{F, m}$ since for this case the population of the lower level 1 (fig. 3 of ref. [17]) is only a fraction of the total $S_{0}$ state population and the excitation terminates most probably in the minimum of the $S_{1}$ potential curve (levels 2 and 3 of fig. 3 in ref. [17] coincide).

The situation of long-wavelength excitation $\nu_{\mathrm{L}}$ $\leqslant \nu_{F, m}$ is displayed in fig. 1. In part (a) a singleminimum potential distribution is depicted which describes our investigated dyes with normal Stokes shifted fluorescence spectra. The presented theory applies also to the saturable absorption of dyes which form excited-state isomers if the time con-

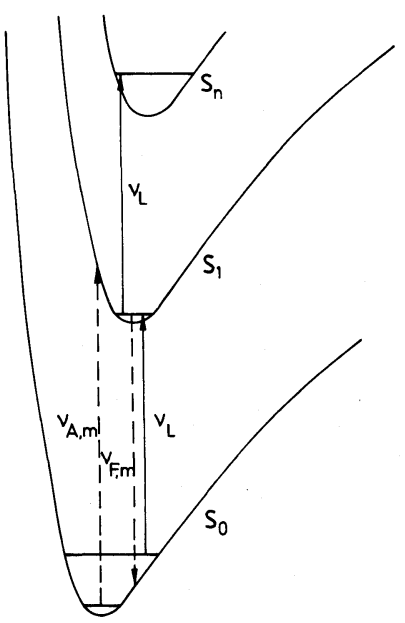

(a)

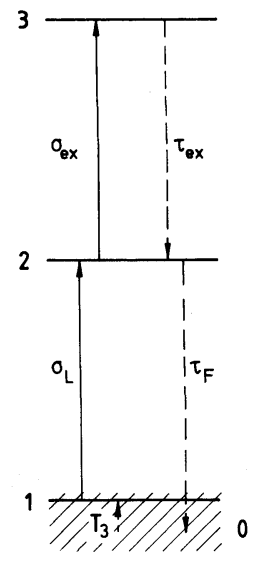

(b)
Fig. 1. (a) Potential-energy diagram. (b) Level system relevant for long-wavelength excitation. stant of isomer formation is long compared to the pump pulse duration. In this case the local $S_{1}$ potential minimum populated within the pump pulse duration would be relevant.

In fig. $1 \mathrm{~b}$ a realistic level system is extracted from the potential curves of fig. 1a. Level 1 indicates the states of molecules (thermally excited and/or in the long-wavelength wing of an inhomogeneous distribution) that take part in the $\mathrm{S}_{0}-\mathrm{S}_{1}$ transition by laser light absorption. Level 2 represents the low-lying states around the $S_{1}$ potential minimum. Level 3 comprises the states in an $S_{n}$ band reached by $S_{1}-S_{n}$ excited-state absorption. Level 0 represents the whole $S_{0}$ band including level 1. The extension of population in level $0\left(\mathrm{~S}_{0}\right.$ band) is indicated by the dashed region. Inclusion of level 1 in level 0 simplifies the calculations. The dynamics of this level system is governed by the following system of differential equations $\left(t^{\prime}=t-\right.$ $\eta z / c ; t$ time; $z$ distance; $\eta$ refractive index; $c$ light velocity in vacuum):

$$
\begin{aligned}
& \frac{\partial N_{1}(\theta)}{\partial t^{\prime}}=-\frac{3 I_{\mathrm{L}}}{h \nu_{\mathrm{L}}} \sigma_{\mathrm{L}} \cos ^{2} \theta\left[N_{1}(\theta)-N_{2}(\theta)\right] \\
&-\frac{N_{1}(\theta)-\bar{N}_{1}}{\tau_{\mathrm{or}}}-\frac{N_{1}(\theta)-\rho_{\mathrm{A}} N_{0}(\theta)}{T_{3}}, \\
& \frac{\partial N_{2}(\theta)}{\partial t^{\prime}}= \frac{I_{\mathrm{L}}}{h \nu_{\mathrm{L}}}\left\{3 \sigma_{\mathrm{L}} \cos ^{2} \theta\left[N_{1}(\theta)-N_{2}(\theta)\right]\right. \\
&\left.-\sigma_{\mathrm{ex}}\left[\mathrm{N}_{2}(\theta)-\mathrm{N}_{3}(\theta)\right]\right\} \\
&-\frac{N_{2}(\theta)}{\tau_{\mathrm{F}}}+\frac{N_{3}(\theta)}{\tau_{\mathrm{ex}}}-\frac{N_{2}(\theta)-\bar{N}_{2}}{\tau_{\mathrm{or}}} \\
& \frac{\partial N_{3}(\theta)}{\partial t^{\prime}=} \frac{I_{\mathrm{L}}}{h \nu_{\mathrm{L}}} \sigma_{\mathrm{ex}}\left[N_{2}(\theta)-N_{3}(\theta)\right] \\
&-\frac{N_{3}(\theta)}{\tau_{\mathrm{ex}}}-\frac{N_{3}(\theta)-\bar{N}_{3}}{\tau_{\mathrm{or}}} \\
& \frac{\partial N_{0}(\theta)}{\partial t^{\prime}}=-\frac{3 I_{\mathrm{L}}}{h \nu_{\mathrm{L}}} \sigma_{\mathrm{L}} \cos ^{2} \theta\left[N_{1}(\theta)-N_{2}(\theta)\right] \\
&+\frac{N_{2}(\theta)}{\tau_{\mathrm{F}}}-\frac{N_{0}(\theta)-\bar{N}_{0}}{\tau_{\mathrm{or}}} \\
& \frac{\partial I_{\mathrm{L}}}{\partial z=-}\left(3 \sigma_{\mathrm{L}} \int_{0}^{\pi / 2}\left[N_{1}(\theta)-N_{2}(\theta)\right]\right. \\
&\left.\times \cos ^{2} \theta \sin \theta \mathrm{d} \theta+\sigma_{\mathrm{ex}} \bar{N}_{2}\right)
\end{aligned}
$$


$\bar{N}_{i}=\int_{0}^{\pi / 2} N_{i}(\theta) \sin \theta \mathrm{d} \theta \quad(i=0,1,2,3)$.

The initial conditions are

$N_{0}\left(\theta, r, t^{\prime}=-\infty, z\right)=N$,

$N_{1}\left(\theta, r, t^{\prime}=-\infty, z\right)=\rho_{\mathrm{A}} N=\alpha\left(\nu_{\mathrm{L}}\right) / \sigma_{\mathrm{L}}$,

$N_{2}\left(\theta, r, t^{\prime}=-\infty, z\right)=N_{3}\left(\theta, r, t^{\prime}=-\infty, z\right)=0$,

and

$I_{\mathrm{L}}\left(r, t^{\prime}, z=0\right)=I_{0 \mathrm{~L}} g(r) f\left(t^{\prime}\right)$.

$N$ is the total number density of dye molecules (molecules $\left./ \mathrm{cm}^{3}\right) . \rho_{\mathrm{A}}=\alpha\left(\nu_{\mathrm{L}}\right) /\left(\sigma_{\mathrm{L}} N\right)$ is the fraction of molecules that interacts with the laser of frequency $\nu_{\mathrm{L}} \cdot \alpha\left(\nu_{\mathrm{L}}\right)\left(\mathrm{cm}^{-1}\right)$ is the absorption coefficient at frequency $\nu_{\mathrm{L}}$ measured with a spectrophotometer. $\sigma_{\mathrm{L}}\left(\mathrm{cm}^{2}\right)$ is the absorption cross section at $\nu_{\mathrm{L}}$. It is identical to the stimulated emission cross section $\sigma_{\mathrm{E}}$ at the same frequency $\nu_{\mathrm{L}}$ which can be determined from the fluorescence spectrum [18] (see below). $g(r)$ and $f\left(t^{\prime}\right)$ are the spatial and temporal pulse profiles, respectively. Gaussian shapes are assumed in the calculations $(g(r)=$ $\left.\exp \left(-r^{2} / r_{0}^{2}\right) ; f\left(t^{\prime}\right)=\exp \left[-\left(t^{\prime}\right)^{2} / t_{0}^{2}\right]\right)$. The energy transmission $T_{\mathrm{E}}$ (ratio of transmitted pulse energy $W_{\mathrm{T}}$ to input energy $W_{\mathrm{I}}$ ) of a laser pulse through the sample is obtained by

$T_{\mathrm{E}}=\frac{\int_{0}^{\infty} \int_{-\infty}^{\infty} I_{\mathrm{L}}\left(r, t^{\prime}, l\right) \mathrm{d} t^{\prime} r \mathrm{~d} r}{I_{0 \mathrm{~L}} \int_{0}^{\infty} g(r) r \mathrm{~d} r \int_{-\infty}^{\infty} f\left(t^{\prime}\right) \mathrm{d} t^{\prime}}$.

The anisotropy of electric dipole interaction is taken into account by $\sigma_{\mathrm{L}}(\theta)=3 \sigma_{\mathrm{L}} \cos ^{2} \theta$. $\theta$ is the angle between direction of electric field strength and direction of the $\mathrm{S}_{0}-\mathrm{S}_{1}$ transition dipole moment. $\bar{N}_{i}(i=0,1,2,3)$ denote orientationally averaged population densities. For the excited-state absorption an average cross section $\sigma_{\mathrm{ex}}$ is used. $\tau_{\mathrm{or}}$ describes the molecular reorientation time. The same $\tau_{\text {or }}$ values are assumed for all levels. Level 1 is depopulated by laser light and it is refilled by thermalization of the $S_{0}$ state distribution (level 0 ). The time constant of repopulation is the spectral cross-relaxation time $T_{3}$.

Excited-state absorption promotes molecules from level 2 to 3 . Molecules in level 3 relax fast to lower states, preferably to level 2 as indicated in fig. $1 \mathrm{~b}$ and eq. (2). The excited-state lifetime $\tau_{\mathrm{ex}}$ is generally very short $[1,2,8]$. A value of $\tau_{\mathrm{ex}}=10^{-13}$ $\mathrm{s}$ is assumed in our calculations. Absorption from level 3 to higher-lying states is taken into account by setting the last term in eq. (5) equal to $\sigma_{\text {ex }} \bar{N}_{2}$ instead of $\sigma_{\text {ex }}\left(\bar{N}_{2}-\bar{N}_{3}\right)$ (open system).

From eq. (2) it is readily seen that the population of the $\mathrm{S}_{1}$ state (level 2) is limited to $N_{2}(\theta) \leqslant$ $N_{1}(\theta) \leqslant \rho_{\mathrm{A}} N /\left(1+\rho_{\mathrm{A}}\right)$ due to the balance of absorption and stimulated emission. No refilling of level 1 within the pump duration $\left(T_{3}>\Delta t_{\mathrm{L}}\right)$ would result in $N_{2}(\theta) \leqslant \rho_{\mathrm{A}} N / 2$ while refilling of level 1 $\left(T_{3}<\Delta t_{\mathrm{L}}\right)$ limits the level 2 population to $N_{2}(\theta) \leqslant$ $\rho_{\mathrm{A}} N /\left(1+\rho_{\mathrm{A}}\right)$. Between level 2 and level 1 no inversion is possible (pulse duration $\Delta t_{\mathrm{L}} \gg$ dephasing time $T_{2}[19]$ ).

For fluorescence emission with $\nu>\nu_{\mathrm{L}}$ the absorption dominates the stimulated emission since the population density in the $S_{0}$ band below level 1 is higher than the population density of level 2 $\left(N_{2}<N_{1}\right)$. This fact hinders amplified spontaneous emission and laser action in the frequency region $\nu>\nu_{\mathrm{L}}$. Laser action and amplified spontaneous emission are limited to the long-wavelength tail of the fluorescence band $\nu<\nu_{\mathrm{L}}$. For a fixed small-signal dye transmission $T_{0}$ at frequency $\nu_{\mathrm{L}}$ the amplified spontaneous emission is reduced since the absorption cross section $\sigma_{\mathrm{A}}\left(\nu_{\mathrm{L}}\right)$ is larger than the emission cross section $\sigma_{\mathrm{E}}(\nu)[17,8]$. Amplified spontaneous emission gains importance with increasing pump laser frequency. In the case of $\nu_{\mathrm{L}} \geqslant \nu_{\mathrm{A}, \mathrm{m}}$, amplified spontaneous emission occurs around the fluorescence emission peak $\nu_{F, m}$. Inversion between $S_{1}$ band and terminating fluorescence Franck-Condon state in $\mathrm{S}_{0}$ band is easily achieved since all molecules take part in the absorption of laser light. For $\nu_{\mathrm{L}} \approx \nu_{\mathrm{A}, \mathrm{m}}$ the cross sections for absorption and stimulated emission are approximately equal $\left[\sigma_{\mathrm{A}}\left(\nu_{\mathrm{A}, \mathrm{m}}\right) \approx \sigma_{\mathrm{E}}\left(\nu_{\mathrm{F}, \mathrm{m}}\right)\right]$. For $\nu_{\mathrm{L}}>\nu_{\mathrm{A}, \mathrm{m}}$ amplified spontaneous emission may become very strong since $\sigma_{\mathrm{E}}\left(\nu_{\mathrm{F}, \mathrm{m}}\right)>\sigma_{\mathrm{A}}\left(\nu_{\mathrm{L}}\right)$. For our experimental situation of $\nu_{\mathrm{L}}<\nu_{\mathrm{F}, \mathrm{m}}$ and $T_{0} \geqslant 0.1$ amplified spontaneous emission is negligible $[17,8]$ and not included in eqs. (1)-(6).

Bleaching of the dye (increase of pulse transmission) is readily achieved for $\nu_{\mathrm{L}}<\nu_{\mathrm{F}, \mathrm{m}}$ as long as the absorption cross section $\left(\sigma_{\mathrm{L}}=\sigma_{\mathrm{E}}\right)$ remains reasonably large because the number of interacting molecules is reduced $\left[\rho_{A}\left(\nu_{L}\right)<1\right]$. At a fixed 
small-signal transmission $T_{0}$ the necessary pulse energy for bleaching should be about the same for $\nu_{\mathrm{L}} \leqslant \nu_{\mathrm{F}, \mathrm{m}}$ and $\nu_{\mathrm{L}} \approx \nu_{\mathrm{A}, \mathrm{m}}$ (same $\sigma_{\mathrm{L}}$ assumed). Bleaching is aggravated for $\nu_{\mathrm{L}}>\nu_{\mathrm{A}, \mathrm{m}}$ because in this frequency range all molecules of the $S_{0}$ state have to be excited to the $S_{1}$ state for complete bleaching $\left(\sigma_{\mathrm{L}}\right.$ reduces with $\left.\nu_{\mathrm{L}}>\nu_{\mathrm{A}, \mathrm{m}}, \rho_{\mathrm{A}}\left(\nu_{\mathrm{L}}\right) \approx 1\right)$

\section{Experimental}

The energy transmission of single picosecond ruby laser pulses $\left(\Delta t_{\mathrm{L}} \approx 25 \mathrm{ps}\right)$ through some dyes has been measured. The laser system and the experimental arrangement are the same as in ref. [20].

Delayed probe pulse transmissions were measured with attenuated ruby laser pulses of perpendicular polarization. An appreciable absorption recovery was not observed up to a delay of $300 \mathrm{ps}$ for the dyes with a fluorescence lifetime in the nanosecond range. A remarkably increased relaxation rate due to amplified spontaneous emission is not expected under our experimental conditions (small number density $N_{2}$, see eqs. (43) and (44) of ref. [17]).

The absorption spectra of the analyzed dyes were measured at a concentration of $2 \times 10^{-6}$ $\mathrm{mol} / \ell$ and at the concentration used in the bleaching experiments. The effective absorption cross sections $\bar{\sigma}_{\mathrm{A}}(\nu)=\rho_{\mathrm{A}}(\nu) \sigma_{\mathrm{A}}(\nu)=\alpha(\nu) / N$ were found to be the same for both concentrations indicating that no dimers were present in the used dye solutions [17].

The fluorescence spectra were recorded by measuring the fluorescence signal under an angle of $90^{\circ}$ to the propagation direction of the excitation light. Very dilute solutions were used $\left(\approx 10^{-6}\right.$ $\mathrm{mol} / \ell$ ) in order to avoid spectral red-shifts due to re-absorption. An unpolarized $2 \mathrm{~mW} \mathrm{He}-\mathrm{Ne}$ laser was used as excitation source. The fluorescence light was dispersed with a $30 \mathrm{~cm}$ spectrograph and registered with a silicon vidicon. The spectra were corrected for the spectral response functions of the spectrograph and the vidicon.

The effective stimulated emission cross section $\bar{\sigma}_{\mathrm{E}}(\nu)=\rho_{\mathrm{E}}(\nu) \sigma_{\mathrm{E}}(\nu)$ is calculated from the $\mathrm{S}_{1}$ radiative lifetime $\tau_{\text {rad }}$ and the spectral shape $E(\nu)$ (quantum distribution, $\int E(\nu) \mathrm{d} \nu=1, \nu$ in $\mathrm{Hz}$ ) of the fluorescence signal according to [18]

$\bar{\sigma}_{\mathrm{E}}(\nu)=c^{2} E(\nu) / 8 \pi \eta^{2} \nu^{2} \tau_{\text {rad }}$.

$\rho_{E}(\nu)$ describes the fraction of molecules interacting with light of frequency $\nu$ in the emission process. It is $\rho_{\mathrm{E}}(\nu) \approx 1$ for $\nu \leqslant \nu_{\mathrm{F}, \mathrm{m}} \cdot \tau_{\text {rad }}$ is calculated by use of the Strickler-Berg formula [21,17]

$\tau_{\mathrm{rad}}^{-1}=\frac{8 \pi \eta^{2}}{c^{2}} \frac{\int E(\nu) \nu^{-1} \mathrm{~d} \nu}{\int E(\nu) \nu^{-4} \mathrm{~d} \nu} \int \bar{\sigma}_{\mathrm{A}}(\nu) \nu^{-1} \mathrm{~d} \nu$

\section{Results and discussion}

The structural formula of the investigated azine dyes (oxazines I-VI; thiazines VII, VIII) and of

I) Oxazine 1

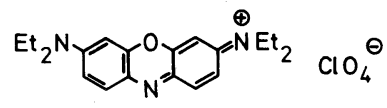

II) Nile blue $A$

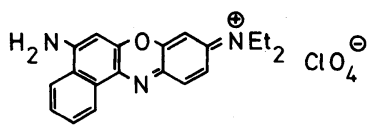

III) Nile blue $\frac{1}{2} \mathrm{SO}_{4}^{\ominus \Theta}$

IV) Oxazine 170

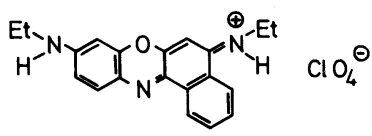

V) Oxazine 4

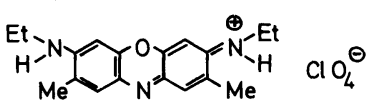

VI) Cresyl violet

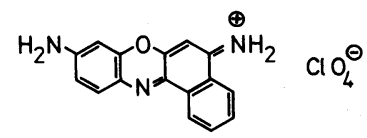

VII) Methylene blue

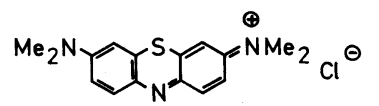

VIII) Toluidine blue 0

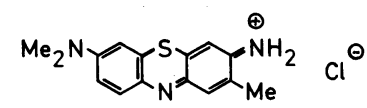

IX) DTDC

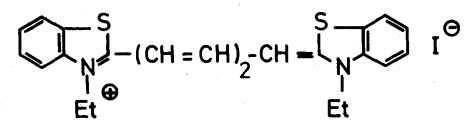

Fig. 2. Structural formulae of investigated dyes. 
Table 1

Data of dyes dissolved in ethanol (analytic grade)

\begin{tabular}{|c|c|c|c|c|c|c|c|c|c|c|c|}
\hline Dye & $\begin{array}{l}\text { Conc. } \\
(\mathrm{mol} / \ell)\end{array}$ & $\begin{array}{l}\lambda_{\mathrm{A}, \mathrm{m}} \\
(\mathrm{nm})\end{array}$ & $\begin{array}{l}\sigma_{\mathrm{A}, \mathrm{m}} \\
\left(\mathrm{cm}^{2}\right)\end{array}$ & $\begin{array}{l}\lambda_{\mathrm{E}, \mathrm{m}} \\
(\mathrm{nm})\end{array}$ & $\begin{array}{l}\sigma_{\mathrm{E}, \mathrm{m}} \\
\left(\mathrm{cm}^{2}\right)\end{array}$ & $\begin{array}{l}\tau_{\text {rad }} \\
\text { (ns) }\end{array}$ & $\begin{array}{l}\tau_{\mathrm{F}} \\
\text { (ns) }\end{array}$ & $T_{0}$ & $\rho_{\mathrm{A}}$ & $\begin{array}{l}\sigma_{\mathrm{L}} \\
\left(\mathrm{cm}^{2}\right)\end{array}$ & $\begin{array}{l}\sigma_{\mathrm{ex}} \\
\left(\mathrm{cm}^{2}\right)\end{array}$ \\
\hline methylene blue & $1.4 \times 10^{-4}$ & 655 & $4 \times 10^{-16}$ & 674 & $2.7 \times 10^{-16}$ & 7.0 & $0.3^{\text {a) }}$ & 0.12 & $5.5 \times 10^{-2}$ & $2.4 \times 10^{-16}$ & $(3 \pm 1)$ \\
\hline oxazine 1 & $5.5 \times 10^{-4}$ & 644 & $4.6 \times 10^{-16}$ & 664 & $4.2 \times 10^{-16}$ & 5.2 & $1.02^{\mathrm{b})}$ & 0.08 & $1.5 \times 10^{-2}$ & $2.5 \times 10^{-16}$ & $(3.5 \pm 1) \times 10^{-17}$ \\
\hline nile blue $\mathrm{A}$ & $4.8 \times 10^{-4}$ & 635 & $2.9 \times 10^{-16}$ & 668 & $3.4 \times 10^{-16}$ & 6.2 & $1.7^{\mathrm{c})}$ & 0.06 & $1.8 \times 10^{-2}$ & $2.6 \times 10^{-16}$ & $(8 \pm 2) \times 10^{-17}$ \\
\hline nile blue & $6.9 \times 10^{-4}$ & 628 & $2.6 \times 10^{-16}$ & 662 & $3.0 \times 10^{-16}$ & 6.8 & $1.9^{\mathrm{d})}$ & 0.12 & $1.0 \times 10^{-2}$ & $1.7 \times 10^{-16}$ & $(7 \pm 1) \times 10^{-17}$ \\
\hline toluidine blue 0 & $7.5 \times 10^{-4}$ & 627 & $1.5 \times 10^{-16}$ & 658 & $1.2 \times 10^{-16}$ & 13.7 & $0.3^{\text {e) }}$ & 0.10 & $3.1 \times 10^{-2}$ & $8.6 \times 10^{-17}$ & $(5 \pm 1) \times 10^{-17}$ \\
\hline oxazine 170 & $1.1 \times 10^{-3}$ & 624 & $3.5 \times 10^{-16}$ & 645 & $4.2 \times 10^{-16}$ & 5.1 & $3.0^{f)}$ & 0.50 & $2.6 \times 10^{-3}$ & $1.5 \times 10^{-16}$ & $(6 \pm 2) \times 10^{-17}$ \\
\hline oxazine 4 & $7.5 \times 10^{-4}$ & 615 & $3.9 \times 10^{-16}$ & 629 & $3.5 \times 10^{-16}$ & 5.1 & $3.2^{\mathrm{g})}$ & 0.12 & $2.1 \times 10^{-2}$ & $1.1 \times 10^{-16}$ & $(1 \pm 0.3) \times 10^{-16}$ \\
\hline cresyl violet & $6.1 \times 10^{-4}$ & 607 & $2.7 \times 10^{-16}$ & 626 & $3.2 \times 10^{-16}$ & 5.9 & $3.51^{\mathrm{b})}$ & 0.18 & $2.8 \times 10^{-2}$ & $8.3 \times 10^{-17}$ & $(7 \pm 1) \times 10^{-17}$ \\
\hline DTDC & $5.1 \times 10^{-5}$ & 655 & $9.5 \times 10^{-16}$ & 678 & $9.7 \times 10^{-16}$ & 3.0 & $1.38^{\mathrm{h})}$ & 0.08 & $5.2 \times 10^{-2}$ & $8.0 \times 10^{-16}$ & $(9 \pm 3) \times 10^{-17}$ \\
\hline
\end{tabular}

a) Fluorescence quantum efficiency $q_{\mathrm{F}}=0.04$ [31]. b) Ref. [32]. c) $q_{\mathrm{F}}=0.27$ [33]. d) Ref. [34].

e) $q_{\mathrm{F}} \approx 0.02$, own measurement. f) $q_{\mathrm{F}}=0.60$ [33]. g) $q_{\mathrm{F}}=0.62$ [33]. h) Ref. [35].

the cyanine dye DTDC (IX) are listed in fig. 2. Characteristic absorption and emission data together with fluorescence lifetimes and experimen-

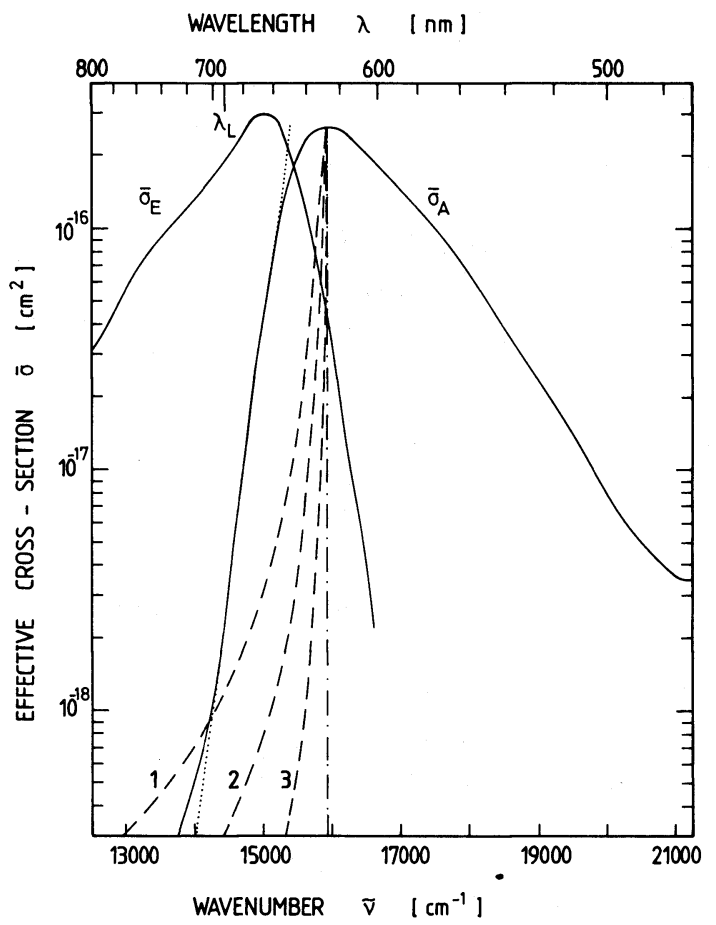

Fig. 3. Absorption and emission spectrum of nile blue sulfate dissolved in ethanol. Dotted line, fitted exponential rise of absorption $\bar{\sigma}_{\mathrm{A}} \propto \exp \left(-\left|\nu-\nu_{\mathrm{A}, \mathrm{m}}\right| h / k T\right)$ with $T=295 \mathrm{~K}$. Dashed curves: low-frequency part of homogeneous line shape centered at $\tilde{\nu}_{\mathrm{A}, \mathrm{m}}=\nu_{\mathrm{A}, \mathrm{m}} / c=15924 \mathrm{~cm}^{-1}$; (1) $\Delta \tilde{\nu}_{\mathrm{H}}=200 \mathrm{~cm}^{-1}$, (2) $\Delta \tilde{\nu}_{\mathrm{H}}=100 \mathrm{~cm}^{-1}$, (3) $\Delta \tilde{\nu}_{\mathrm{H}}=40 \mathrm{~cm}^{-1}$.

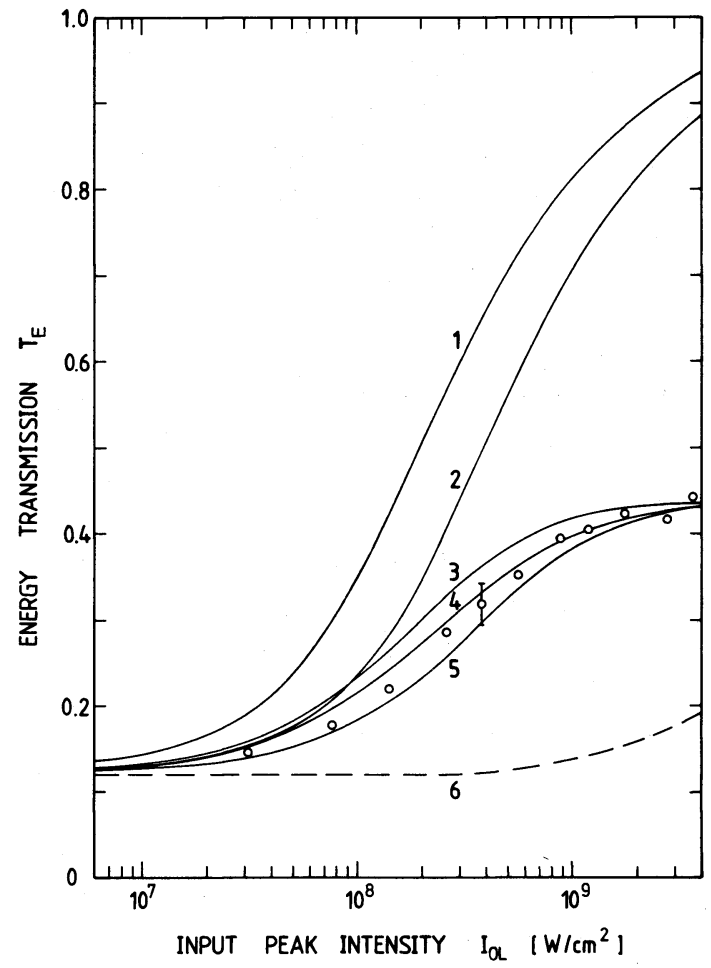

Fig. 4. Energy transmission versus input peak intensity. Dye nile blue sulfate dissolved in ethanol. Cell length $3 \mathrm{~cm}$. Reorientation time $\tau_{\mathrm{or}}=220$ ps [34]. Pulse duration $\Delta t_{\mathrm{L}}=25 \mathrm{ps}$ (fwhm). Curves are calculated for (1) $\sigma_{\mathrm{ex}}=0$ and $T_{3}=\infty$; (2) $\sigma_{\mathrm{ex}}=0$ and $T_{3} \ll \Delta t_{\mathrm{L}} ;$ (3) $\sigma_{\mathrm{ex}}=1.4 \times 10^{-16} \mathrm{~cm}^{2}$ and $T_{3}=\infty$, (4) $\sigma_{\text {ex }}=10^{-16} \mathrm{~cm}^{2}$ and $T_{3}=\Delta t_{\mathrm{L}}$, (5) $\sigma_{\mathrm{ex}}=7 \times 10^{-17} \mathrm{~cm}^{2}$ and $T_{3} \ll \Delta t_{\mathrm{L}}$. Dashed curve, $\rho_{\mathrm{A}}=1, \sigma_{\mathrm{L}}=\bar{\sigma}_{\mathrm{L}}=\alpha_{\mathrm{L}} / N=1.7 \times 10^{-18}$ $\mathrm{cm}^{2}, \sigma_{\mathrm{ex}}=7 \times 10^{-19} \mathrm{~cm}^{2}$; eqs. (2)-(6) are used with $N_{1}(\theta)=$ $N_{0}(\theta)$. Other dye parameters are listed in table 1 . 
tally determined excited-state absorption cross sections are listed in table 1 . As an example we give detailed curves for the dye nile blue sulfate dissolved in ethanol in the following.

The effective absorption spectrum $\bar{\sigma}_{\mathrm{A}}(\lambda)=$ $\alpha(\lambda) / N$ and the effective emission spectrum $\bar{\sigma}_{\mathrm{E}}(\lambda)$ are displayed in fig. 3 . The position of the laser wavelength $\lambda_{\mathrm{L}}$ is indicated.

The energy transmission $T_{\mathrm{E}}$ versus input peak intensity $I_{\mathrm{OL}}$ is plotted in fig. 4. Experimental points together with calculated curves are shown. Curves 1 and 2 are calculated for $T_{3}=\infty$ and $T_{3} \ll \Delta t_{\mathrm{L}}$ [eq. (1) replaced by $N_{1}\left(\theta, t^{\prime}\right)=$ $\left.\rho_{\mathrm{A}} N_{0}\left(\theta, t^{\prime}\right)\right]$, respectively. The excited-state absorption cross section is set to $\sigma_{\mathrm{ex}}=0$. A factor of 2 higher pulse intensity is needed for $T_{3} \ll \Delta t_{\mathrm{L}}$ compared to $T_{3}=\infty$ in order to bleach the dye to the same energy transmission. The refilling of level 1 within the pump pulse duration in case of $T_{3} \ll \Delta t_{\mathrm{L}}$ is responsible for the reduced bleaching. The excited-state absorption cross section $\sigma_{\mathrm{ex}}$ is obtained by fitting the theoretical curves to the experimental points. The $\sigma_{\text {ex }}$ value depends on the cross-relaxation time $T_{3}$ since for $T_{3} \gg \Delta t_{\mathrm{L}}$ the number density $N_{2}\left(I_{0 \mathrm{~L}} \rightarrow \infty\right) \approx \rho_{\mathrm{A}}\left(\nu_{\mathrm{L}}\right) N / 2$ is approximating a factor of two smaller than for $T_{3} \ll \Delta t_{\mathrm{L}}$, where the population of level 2 approaches $N_{2}\left(I_{0 \mathrm{~L}}\right.$ $\rightarrow \infty) \approx \rho_{\mathrm{A}}\left(\nu_{\mathrm{L}}\right) N /\left(1+\rho_{\mathrm{A}}\right)$ (see above). The curves $3\left(T_{3}=\infty, \sigma_{\mathrm{ex}}=1.4 \times 10^{-16} \mathrm{~cm}^{2}\right), 4\left(T_{3}=\Delta t_{\mathrm{L}}=25\right.$ ps, $\left.\sigma_{\text {ex }}=10^{-16} \mathrm{~cm}^{2}\right)$ and $5\left(T_{3} \ll \Delta t_{\mathrm{L}}, \sigma_{\mathrm{ex}}=7 \times\right.$ $10^{-17} \mathrm{~cm}^{2}$ ) clearly demonstrate this fact. They fit to the experimental energy transmission at high input peak intensities.

The spectral cross-relaxation $T_{3}$ has to be longer than half the dephasing time $T_{2}\left(T_{3} \geqslant T_{2} / 2\right)$, since $T_{3}$ contributes to $T_{2}\left(T_{2}^{-1}=\left(2 T_{3}\right)^{-1}+\left(T_{2}^{\prime}\right)^{-1} ; T_{2}\right.$ phase relaxation time of level $1 ; T_{2}^{\prime}$ pure dephasing time of level $1 ; T_{3}$ energy relaxation time of level 1 , here specially spectral cross-relaxation time). A lower limit of the dephasing time $T_{2}$ may be estimated from the spectral shape of the absorption spectrum in the long-wavelength tail. The shape of the absorption spectrum generally is determined by a convolution of transitions between rovibrational levels in the $S_{0}$ and $S_{1}$ band, by thermal level population, by inhomogeneous $S_{0}-S_{1}$ transition spacing due to irregular solute-solvent interaction, and by homogeneous broadening.
The spectral shape of the absorption spectrum in the long-wavelength wing is sometimes governed by thermal level population. The dotted line in fig. 3 indicates the expected rise of absorption due to thermal level population $\left[\bar{\sigma}_{\mathrm{A}}(\nu) \propto \exp (-\mid \nu-\right.$ $\left.\left.\nu_{\mathrm{A}, \mathrm{m}} \mid h / k T\right)\right]$ at $T=295 \mathrm{~K}$. The slope of the absorption curve could not be fitted for all investigated substances to the experimental room temperature (e.g. fitted temperature is $250 \mathrm{~K}$ in case of oxazine 1 and $370 \mathrm{~K}$ in case of toluidine blue 0 ) indicating influences of inhomogeneous broadening and Franck-Condon factors. Out in the long-wavelength wing the absorption cross section decays more slowly than expected from thermal population (exponential decrease) or inhomogeneous origin (gaussian decrease). This fact is seen by the deviation of the $\bar{\sigma}_{\mathrm{A}}$ curve from the dotted line in fig. 3. The reduced decrease of $\bar{\sigma}_{\mathrm{A}}$ is most probably due to homogeneous broadening (lorentzian shape). Contributions of triplet absorption and dimer absorption cannot be completely excluded.

Three homogeneous line shapes of homogeneous linewidth $\Delta \tilde{\nu}_{\mathrm{H}}=200 \mathrm{~cm}^{-1}$ (curve 1), $\Delta \tilde{\nu}_{\mathrm{H}}=$ $100 \mathrm{~cm}^{-1}$ (curve 2) and $\Delta \tilde{\nu}_{\mathrm{H}}=40 \mathrm{~cm}^{-1}$ (curve 3) are included in fig. 3 . The shape of curve $3\left(\Delta \tilde{\nu}_{H}\right.$ $=40 \mathrm{~cm}^{-1}$ ) fits the shape of the absorption spectrum in the very-low-frequency wing. Since other mechanisms might contribute to the absorption spectrum in this region, $\Delta \tilde{\nu}_{\mathrm{H}}=40 \mathrm{~cm}^{-1}$ gives only an upper limit of the homogeneous linewidth.

The homogeneous linewidth $\Delta \tilde{\nu}_{\mathrm{H}}(\mathrm{fwhm})$ is related to the dephasing time by $T_{2}=1 / \pi c \Delta \nu_{\mathrm{H}}$ $[22,30]$. In the case of nile blue sulfate dissolved in ethanol we estimate $T_{2} \geq 2.5 \times 10^{-13} \mathrm{~s}\left(T_{3}>T_{2} / 2\right)$. Reported $T_{2}$ values of organic dye solutions at room temperature are in the same time region (1,1'-diethyl-4,4'-quinocyanine iodide in ethanol: $T_{2}=0.2-0.6$ ps [23]; cryptocyanine in methanol: $T_{2}=0.6-1$ ps [25] and $0.1-1$ ps [26], DDI in glycerin: $T_{2} \approx 0.4 \pm 0.2$ ps [27]).

The energy transmission data also exclude a complete homogeneous transition [28-30]. In this case all molecules would take part in the absorption even at $\nu_{\mathrm{L}}<\nu_{\mathrm{F}, \mathrm{m}}\left[\rho_{\mathrm{A}}\left(\nu_{\mathrm{L}}\right)=1\right]$ and the nonlinear transmission should follow the dashed curve in fig. 4. The Rayleigh-induced optical Kerr-effect polarization results of ref. [28] might result from 
spectral diffusion of excited molecules which extends the induced dichroism and birefringence over the whole inhomogeneously broadened band [29].

\section{Conclusions}

The dynamics of some dyes excited to the longwavelength region of the $S_{0}-S_{1}$ absorption band has been investigated. The $S_{0}-S_{1}$ excitation is believed to terminate in the potential minimum of the $S_{1}$ state (Franck-Condon state and temporal equilibrium state coincide). As a consequence no inversion is achievable for the region of optimum stimulated emission cross section $\left(\nu>\nu_{\mathrm{L}}\right)$. Amplified spontaneous emission and net gain in a laser resonator are limited to the long-wavelength tail of the fluorescence band (reduced efficiency due to decreasing $\sigma_{\mathrm{E}}$ for $\nu<\nu_{\mathrm{L}}$ ). Bleaching of dyes is readily observed and the application of saturable absorbers may be extended to the long-wavelength region of the $S_{0}-S_{1}$ transition [37,38].

\section{Acknowledgement}

The authors thank Th. Ascherl for technical assistance and the Rechenzentrum of the University for disposal of computer time.

\section{References}

[1] C.V. Shank, E.P. Ippen and O. Teschke, Chem. Phys. Letters 45 (1977) 291.

[2] W. Falkenstein, A. Penzkofer and W. Kaiser, Opt. Commun. 27 (1978) 151.

[3] A.H. Zewail, in: Springer Series in Chemical Physics, Vol. 23. Picosecond phenomena, Part III, eds. K.B. Eisenthal, R.M. Hochstrasser, W. Kaiser and A. Laubereau (Springer, Berlin, 1982) p. 184.

[4] C.S. Parmenter and K.Y. Tang, Chem. Phys. 27 (1978) 127.

[5] V. Sundström, T. Gillbro and H. Bergström, Chem. Phys. 73 (1982) 439.

[6] H. Staerk, R. Mitzkus, W. Kühnle and A. Weller, in: Springer Series in Chemical Physics, Vol. 23 Picosecond phenomena, Part III, eds. K.B. Eisenthal, R.M. Hochstrasser, W. Kaiser and A. Laubereau (Springer, Berlin, 1982) p. 205.
[7] M.E. Mack, Appl. Phys. Letters 15 (1969) 166

[8] A. Penzkofer and W. Falkenstein, Opt. Quantum Electron. 10 (1978) 399.

[9] A. Müller, J. Schulz-Henning and H. Tashiro, Appl. Phys. 12 (1977) 333.

[10] J. Wiedmann and A. Penzkofer, Nuovo Cimento 63B (1981) 459.

[11] S.L. Chin and A. Zardecki, Phys. Rev. A13 (1976) 1528.

[12] S.L. Chin and D. Belanger, Opt. Commun. 16 (1976) 121.

[13] D. Faubert, S.L. Chin, M. Cormier and M. Boloten, Can. J. Phys. 57 (1979) 160.

[14] D. Faubert and S.L. Chin, Can. J. Phys. 57 (1979) 1359.

[15] D. Faubert and S.L. Chin, Can. J. Phys. 60 (1982) 261.

[16] D. Leupold, R. König, B. Voigt and R. Menzel, Opt. Commun. 11 (1974) 78.

[17] A. Penzkofer and W. Blau, Opt. Quantum Electron. 15 (1983) 325.

[18] O.G. Peterson, J.P. Webb, W.C. McColgin and J.H. Eberly, J. Appl. Phys. 42 (1971) 1917.

[19] A. Yariv, Quantum electronics, 2nd Ed. (Wiley, New York, 1975) ch. 15.

[20] W. Blau, R. Reber and A. Penzkofer, Opt. Commun. 43 (1982) 210.

[21] S.J. Strickler and R.A. Berg, J. Chem. Phys. 37 (1962) 814.

[22] M.D. Levenson, Introduction to nonlinear laser spectroscopy (Academic Press, New York, 1982).

[23] T. Yajima, H. Souma and Y. Ishida, Phys. Rev. A17 (1978) 324.

[24] H. Souma, T. Yajima and Y. Taira, J. Phys. Soc. Japan 48 (1980) 2040.

[25] G. Mourou, IEEE J. Quantum Electron. QE-11 (1975) 1.

[26] L. Huff and L.G. DeShazer, J. Opt. Soc. Am. 60 (1970) 157.

[27] D.W. Vahey and A. Yariv, Phys. Rev. A10 (1974) 1578.

[28] J.J. Song, J.H. Lee and M.D. Levenson, Phys. Rev. A17 (1978) 1439.

[29] Y. Taira and T. Yajima, J. Phys. Soc. Japan 50 (1981) 3459.

[30] A. Penzkofer, A. Laubereau and W. Kaiser, Progr. Quantum Electron. 6 (1979) 55.

[31] J. Olmsted III, J. Phys. Chem. 83 (1979) 2581.

[32] G.S. Beddard, T. Doust and G. Porter, Chem. Phys. 61 (1981) 17.

[33] R. Sens and K.H. Drexhage, J. Luminescence 24/25 (1981) 709.

[34] H.E. Lessing and A. von Jena, in: Laser handbook, Vol. 3, ed. M.L. Stitch (North-Holland, Amsterdam, 1979) p. 784.

[35] W. Sibbett, J.R. Taylor and D. Welford, IEEE J. Quantum Electron. QE-17 (1981) 500.

[36] M.W. McGeoch, Opt. Commun. 7 (1973) 116.

[37] E.G. Arthurs, D.J. Bradley, P.N. Puntambekar, I.S. Ruddock and T.J. Glynn, Opt. Commun. 12 (1974) 360. 